\title{
Self-Organization-Based Fabrication of Stable Noble-Metal Nanostructures on Large-Area Dielectric Substrates
}

\author{
Victor Ovchinnikov ${ }^{1}$ and Andriy Shevchenko \\ ${ }^{1}$ School of Electrical Engineering, Aalto Nanofab, Aalto University, P.O. Box 13500, 00076 Aalto, Finland \\ ${ }^{2}$ Department of Applied Physics, School of Science, Aalto University, P.O. Box 13500, 00076 Aalto, Finland \\ Correspondence should be addressed to Victor Ovchinnikov; victor.ovchinnikov@aalto.fi
}

Received 15 January 2013; Accepted 28 February 2013

Academic Editor: Pietro Calandra

Copyright (c) 2013 V. Ovchinnikov and A. Shevchenko. This is an open access article distributed under the Creative Commons Attribution License, which permits unrestricted use, distribution, and reproduction in any medium, provided the original work is properly cited.

\begin{abstract}
A cost-effective fabrication of random noble-metal nanostructures with a feature size of the order of $10 \mathrm{~nm}$ on a large-area dielectric substrate is described. The method combines dry etching of the substrate through a self-organized metal mask with a directional deposition of a multilayered metal film. The technique allows one to create metal nanoislands on a nanopatterned dielectric template with an enhanced adhesion between the metal and the dielectric. The use of the adhesion layer-that makes the structures stableis important in view of variety of optical and other potential applications of the structures. We observe that the presence of the adhesion sublayer dramatically influences both the morphological and optical properties of the structures. The results of this work can be of interest in regard to the development of new approaches to self-organization-based nanofabrication of extremely small metal and metal-dielectric nanostructures on large-area substrates.
\end{abstract}

\section{Introduction}

Metal nanostructures have widespread applications in many areas of science and technology, such as chemical and biological sensing [1], subwavelength imaging [2], and plasmonic nanomaterials [3]. The majority of these applications rely on optical excitation of plasmon resonances in metal nanoparticles that can be either isolated or arranged in a nanoparticle array. Spectral properties of these resonances can be adjusted by tailoring the size, shape, and separation distance of the nanoparticles. The well-studied periodic nanopatricle arrays, which, in particular, are very attractive from a theoretical point of view, are difficult to fabricate on large-area substrates and, therefore, their mass production is a challenging task. Traditional semiconductor technology offers reliable and reproducible process flows, but their acceptance as a general fabrication tool for obtaining large-area metal nanostructures has been hindered by two drawbacks: (1) a limited resolution of optical lithography and (2) a lack of efficient dry etching techniques for most metals. Because of this, a dominant fabrication method for obtaining regular metal nanostructures is electron beam lithography (EBL) with lift-off removal of useless metal. However, by using this technique, it is difficult to obtain features smaller than $50 \mathrm{~nm}$. In addition, the fabrication approach based on EBL requires a rather long processing time, which makes it not only timeconsuming, but also expensive to fabricate large-area arrays of nanostructures.

As alternatives to step-by-step patterning by conventional EBL, several nonlithographic methods-for example, based on self-organization phenomenon-have been demonstrated to produce nanostructures in parallel way. These techniques allow large-area substrates to be nanostructured using fast and low-cost fabrication steps. The functional properties of the nanostructures, however, are less controllable due to the inherent randomness in the size, shape, and spacing of the created particles. In spite of this, random nanostructure arrays are widely used, for example, as catalysts in fabrication of carbon nanotubes [4], light trapping layers in thin-film solar cells [5,6], magnetic nanodot arrays [7], SERS substrates [8], and environment sensors [9]. Examples of fast and cost-effective nonlithographic techniques are nanosphere lithography [10], nanofabrication using porous alumina templates [11] and so-called oblique angle deposition [12]. 
One of the fastest and most cost-effective nanofabrication approaches is an approach making use of self-assembly of metal nanostructures on a surface with poor adhesion, for example, Au or Ag nanoislands on glass [13, 14]. This method possesses high resolution and, what is practically important, full compatibility with standard semiconductor fabrication technology. By the nature of the phenomenon, self-organization takes place in a thin layer of noble metal on a dielectric substrate at nonequilibrium thermodynamic conditions, when the adhesion of the metal to the substrate is weak. Such self-organized nanoparticle arrays are not mechanically stable and can easily be damaged-for example, rinsing the sample in water can completely destroy the array. Hence, such arrays are nearly impossible to incorporate in more complicated, multilayered devices.

In this work, a self-organization-based approach to nanofabrication of large-area arrays of metal nanostructures on a dielectric substrate with an adhesion sublayer is demonstrated. This is done by creating identical lace-like $\mathrm{Au}$ and $\mathrm{Ag}$ nanostructures with and without $\mathrm{Ti}$ sublayer on glass and oxidized silicon substrates. In particular, these nanostructures allow us to directly study the influence of the adhesion layer on the morphology and optical properties of the samples. We note that noble metal nanostructures without an adhesion sublayer cannot be created by using a lift-offbased fabrication method, such as EBL, and, consequently, direct estimation of the influence of such a layer on the optical properties of the sample is impossible; some experimental studies of the influence of the adhesion-layer thickness on the properties of a flat metal film can be found in [15-17].

In the following section, we introduce our fabrication method and describe in detail the sample preparation and measurement procedures. The next section consists of two subsections. In the first one, the morphology of the fabricated nanostructures is studied on the basis of SEM images of the samples obtained at different deposition conditions. The second subsection describes the transmittance, reflectance, absorptance, and SERS spectra of these samples. The last section summarizes our results.

\section{Materials and Methods}

2.1. Fabrication Method. Our fabrication method is based on (i) self-assembly of metal nanostructures on a dielectric substrate, (ii) fabrication of a nanostructured template by etching the substrate through the self-organized metal mask, and (iii) directional deposition of several different metal layers on the obtained template. In principle, for the template preparation, any nanopatterning method that provides vertical sidewalls can be used (e.g., etching through lithographically fabricated masks or self-organized structures and even direct writing by a focused laser or ion beam $[18,19])$.

We start by creating a self-organized gold or silver nanostructures which are then used as a mask for anisotropic dry etching of the substrate (Figure 1(a)). To create the mask, a thin layer of gold or silver is evaporated onto an oxidized silicon or glass substrate at a low deposition rate. If the layer thickness does not exceed $15 \mathrm{~nm}$, the film made of gold shows a lace-like structure owing to the poor adhesion of the metal to silicon oxide. An additional annealing after the deposition can be employed to modify the mask morphology. In contrast to gold, silver tends to form spheroidal nanoislands with the average size and separation controlled by the deposition and annealing parameters [6].

The poor adhesion of gold and silver to the dielectrics limits the etching methods for the substrate patterning. Wet etching cannot be used, because of destructive surfacetension forces and diffusion of the liquid etchant beneath the metal. Dry etching, on the other hand, can be successfully used. In addition, dry etching, such as reactive ion etching (RIE), provides vertical sidewalls for the nanostructures. Therefore we use RIE for etching the dielectric through the obtained mask (Figure 1(b)).

In the next step, the residues of the metal mask are removed by selective wet etching to obtain a template that is made of the substrate material only (see Figure 1(c)). The template elements perfectly replicate the geometry of the mask. Then the template is covered with a thin adhesion layer of an easily oxidized metal (such as $\mathrm{Ti}$ or $\mathrm{Cr}$ ). At atmospheric ambient conditions, the adhesion layer that is not protected by any other material layer is readily oxidized and does not affect much the functional properties of the nanostructures. It is important to deposit the adhesion layer only on the flat (horizontal) surfaces of the template and leave the sidewalls uncovered (Figures $1(\mathrm{~d})-1(\mathrm{f})$ ). This helps one to avoid unwanted deposition of the next metal layer onto the sidewalls and, thus, to better preserve the desired nanostructure geometry. This requirement is fulfilled by using a directional flux of deposited material, for example, ebeam evaporation at normal incidence. Even if some noblemetal particles turn out to be deposited onto the sidewalls, they can easily be removed, owing to their poor adhesion, by rinsing the sample in an ultrasonic bath.

The final step is to coat the template with a functional metal layer-with a gold or silver layer in our case. This should be done immediately after the deposition of the adhesion layer in order to prevent possible oxidation of the latter. This metal coating can be done in two different ways. In the first one, the metal is evaporated onto the wafer surface at normal incidence. Therefore, a metal layer is formed simultaneously on the elevated surfaces of the nanostructure and at the bottom of the template (Figure $1(\mathrm{~d})$ ). The second way is to evaporate the metal onto the template at an angle. The sample can be either rotated (Figure 1(f)) or kept still during the deposition (Figure 1(e)). The rotation provides a uniform covering of the structure upper parts, while the static deposition leads to formation of asymmetric nanostructures that, for example, can exhibit optical anisotropy [20]. The extent to which the sidewalls are covered with metal depends significantly on the evaporation angle $\alpha$ counted from the normal of the substrate surface (Figure 1(e)) [21].

2.1.1. Experimental Details. The samples were fabricated either on borosilicate glass plates or on oxidized silicon substrates with (100)-orientation. The sample size is $22 \times$ $22 \times 0.5 \mathrm{~mm}^{3}$. The substrate cleaning procedure starts with 


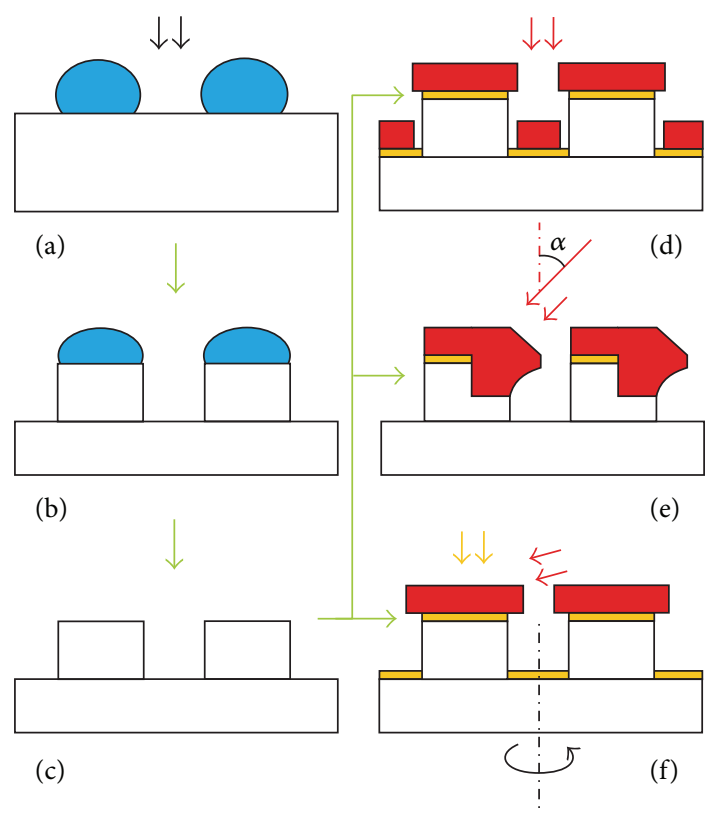

FIGURE 1: Schematic of the process of self-organization-based nanofabrication of stable noble-metal nanostructures on a dielectric substrate. The process consists of four steps. The fourth step is chosen to correspond to either (d), (e), or (f).

sonification of the sample in acetone and 2-propanol. Then the sample is RCA-1 cleaned for 10 minutes and processed in oxygen plasma during 1 minute. This pretreatment is needed to equalize the surface conditions over the whole substrate. The $\mathrm{Si}$ samples with a $60 \mathrm{~nm}$ thick layer of $\mathrm{SiO}_{2}$ were used to optimize the whole fabrication process, since they make it possible to observe the fabricated nanostructures with SEM. We note that all the process steps lead to identical results on glass and on silicon oxide.

The metal films were deposited with the help of an e-beam evaporation system IM-9912 (Instrumentti Mattila Oy) with adjustable substrate inclination and rotation speed at the base pressure of $4 \times 10^{-7}$ Torr at room temperature. The deposition rate for each film was measured by using quartz crystal microbalance.

The RIE of the templates was done in a $13.56 \mathrm{MHz}$ driven parallel electrode reactor Plasmalab 80 Plus (Oxford Instruments Plasma Technology). An anisotropic etching process based on fluorine chemistry (gas mixture $\mathrm{CF}_{4}: \mathrm{CHF}_{3}$ $=1: 3$ ) was used for the glass and $\mathrm{SiO}_{2}$ etching. The etching experiments were performed at a total gas flow of $80 \mathrm{sccm}$, pressure of 30 mTorr and rf power of $40 \mathrm{~W}$. The remaining gold was removed in aqua regia (1:3 volume mixture of $69 \%$ $\mathrm{HNO}_{3}$ and $37 \% \mathrm{HCl}$, resp.) during $20 \mathrm{~s}$ at room temperature, resulting in lace-like structures in $\mathrm{SiO}_{2}$ or glass. Mask annealing was done at $400 \mathrm{C}$ during $20 \mathrm{~min}$ in $\mathrm{N}_{2}$ ambient.

SEM images of the fabricated samples were taken with Zeiss Supra 40 field emission scanning electron microscope. The transmittance and reflectance spectra of the samples were measured at normal incidence by using PerkinElmer Lambda 950 UV-VIS spectrometer in the spectral range from 300 to $850 \mathrm{~nm}$. The reflectance spectra were measured by collecting the directly reflected and backscattered light with an integrating-sphere detector. The surface enhanced Raman scattering (SERS) of the molecules of methylene blue (MB) on the fabricated substrates was studied by using a WITec Alpha 300 Raman microscope equipped with a frequencydoubled Nd:YAG laser $(532 \mathrm{~nm}$ ) as the excitation source. A water solution of methylene blue at a concentration of $3 \times$ $10^{-4} \mathrm{M}$ was used as a test analyte.

\section{Results and Discussion}

3.1. SEM Observations. To demonstrate the performance of the method described in Section 2, we have fabricated gold and silver lace-like nanostructures. In order to study the effect of an adhesion layer beneath the metal, some of the samples were fabricated by directly metal-coating the patterned $\mathrm{SiO}_{2}$ or glass surface and some other samples included an additional adhesion sublayer of Ti. Such mechanically stable noble-metal nanostructures are of interest in view of variety of nanooptical applications. Figures 2-7 illustrate the most essential steps of practical realization of the method depicted in Figure 1. As an etching mask (Figure 1(a)), we use the nanostructures formed on a dielectric surface during the physical vapor deposition of gold or silver, although other metals with poor adhesion to the substrate could also be used.

The shape and size of the lace-like $\mathrm{Au}$ nanostructures can be controlled by varying the fabrication parameters and conditions at each step of the process. It is important to notice that the main features of the nanopattern to be fabricated are defined already at the initial metal deposition stage. Figures 2 and 3 show self-organized metal nanostructures produced by coating an oxidized silicon surface with gold and silver, respectively, using the e-beam evaporation. SEM images of the samples with four different thicknesses of the coating were taken. The metals are observed to produce a variety of the nanostructure geometries with different size distributions. For both metals, the thicker the deposited layer, the larger the fraction of the metal-covered area and the sizes of the eventually obtained nanofeatures. The difference between the patterns made of gold and silver is significant. In the case of gold, continuous net- or lace-like structures with narrow channels are observed, while when using silver, wellseparated nanoparticles of various shapes and sizes appear to cover the whole surface of the substrate. The geometry of such metal masks depends on many factors, such as surface diffusion characteristics, the flux of the deposited material, and the concentration of the nucleation centers on the surface. In this context, the film thickness is the most essential adjustable parameter that can be used to control the structure geometry. For example, a $15 \mathrm{~nm}$ thick gold film shown in Figure 2(d) exhibits smaller widths and a lower density of the empty channels than a $10 \mathrm{~nm}$ thick film deposited at the same deposition conditions (see Figure 2(b)). A $4 \mathrm{~nm}$ thick silver film (Figure 3(a)), on the other hand, shows approximately six times higher density of the nanoislands than a $12 \mathrm{~nm}$ thick film (Figure 3(d)). The maximum obtainable average size of the nanoislands is limited by their coalescence and transformation into a continuous film. When dealing with gold and 

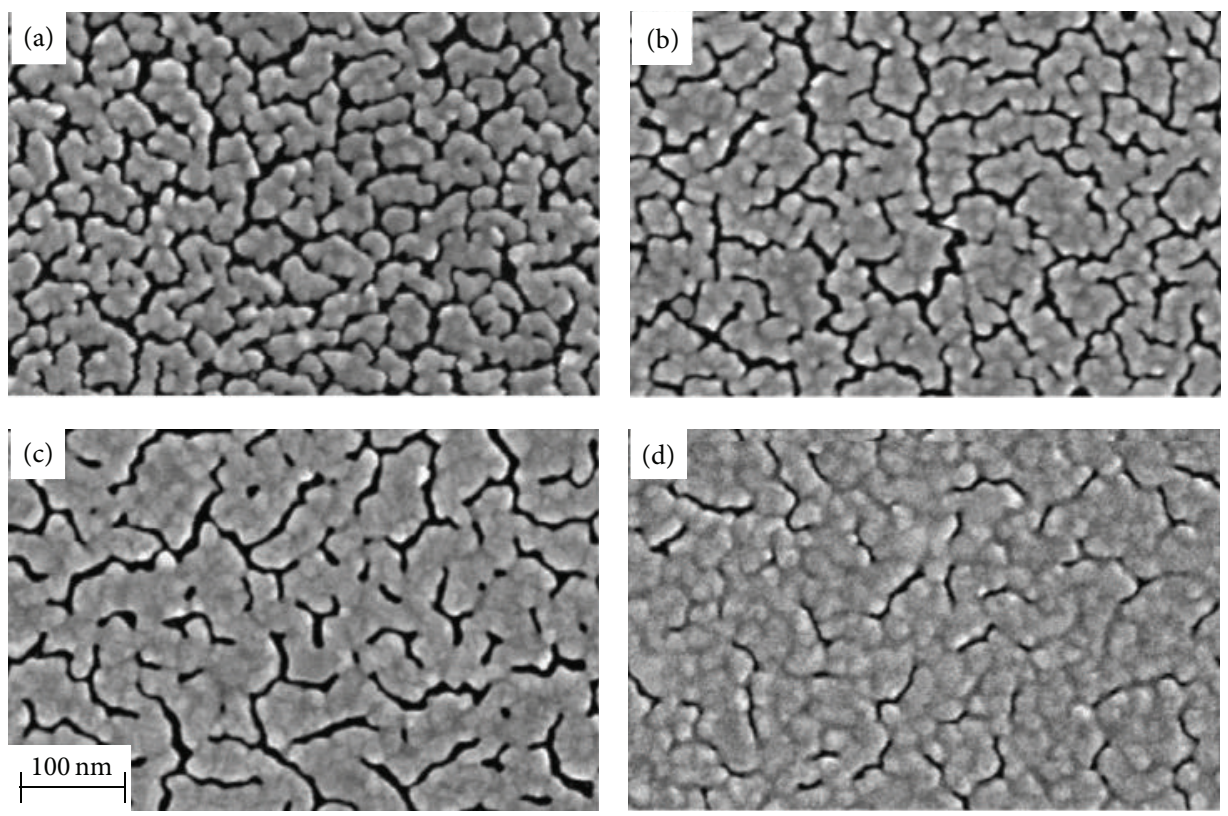

Figure 2: SEM images of Au films directly deposited on a flat dielectric substrate. The film thickness is 8, 10, 11, and 15 nm in (a), (b), (c), and (d), respectively.
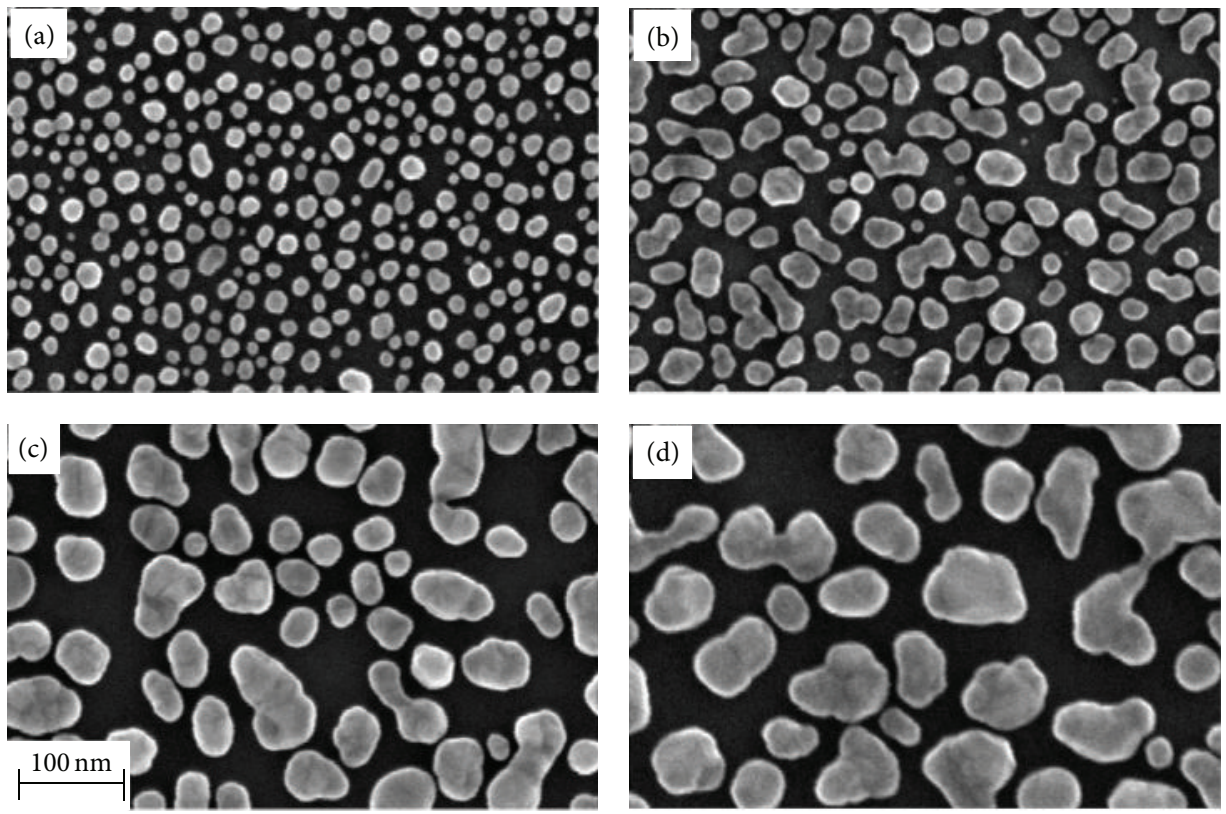

FIGURE 3: SEM images of Ag films directly deposited on a dielectric substrate. The film thickness is 4, 5.5, 10, and 12 nm in (a), (b), (c), and (d), respectively.

silver, this transformation takes place if the film thickness exceeds $20 \mathrm{~nm}$. An additional control of the mask pattern geometry can be provided by changing the metal deposition rate, substrate temperature, and surface properties.

Compared to well-known self-organized $\mathrm{Au}$ and $\mathrm{Ag}$ masks [22], the masks created in the present study do not require any annealing. The directly deposited self-organized films are used in their original form, which allows keeping the feature sizes small. On the other hand, annealing is an excellent tool for further shaping and sizing of the created metal pattern. Due to combined effects of a weak adhesion force, increased surface diffusion, and thermal stresses, the structures can be fully rearranged during the annealing. Figure 4 provides a direct comparison of the pattern 

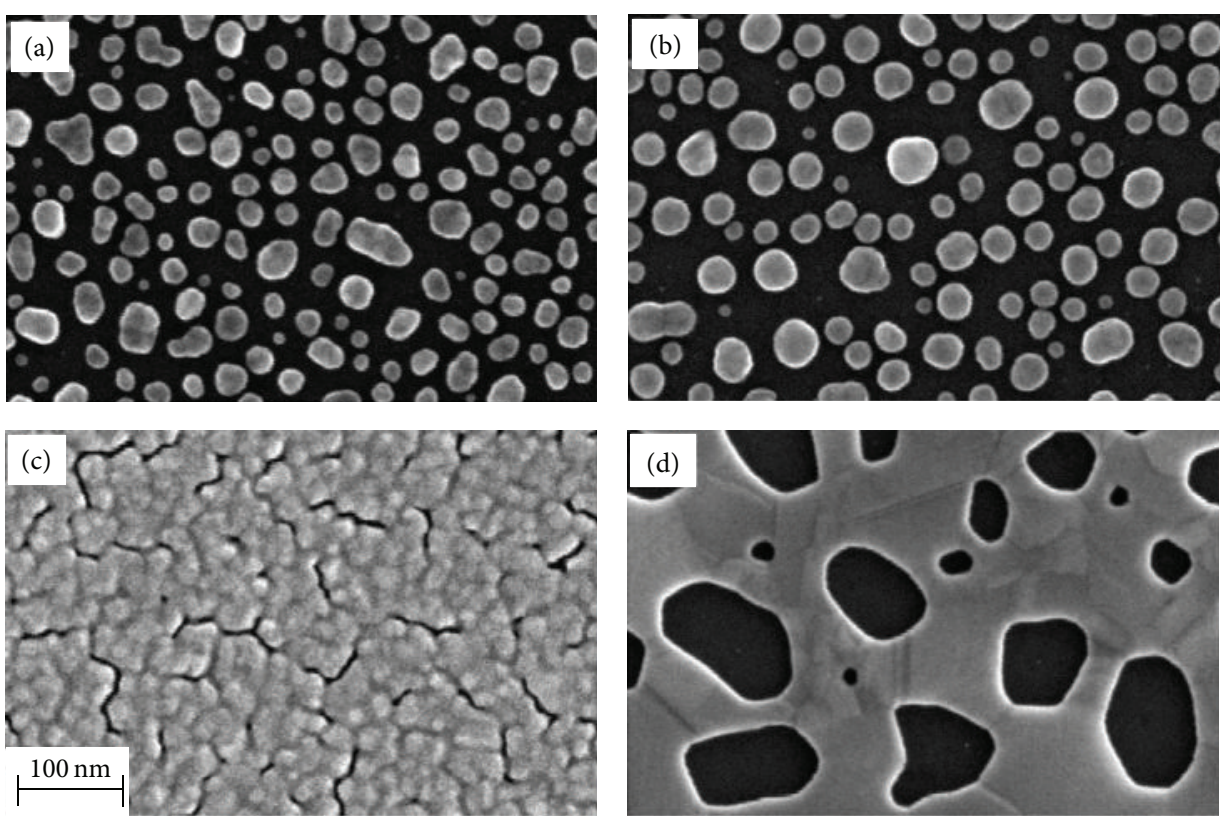

Figure 4: SEM images of thin layers of Ag (cases (a) and (b)) and Au ((c) and (d)) before ((a) and (c)) and after ((b) and (d)) annealing at $400^{\circ} \mathrm{C}$. The thicknesses of $\mathrm{Ag}$ and Au layers are 5 and $15 \mathrm{~nm}$, respectively.
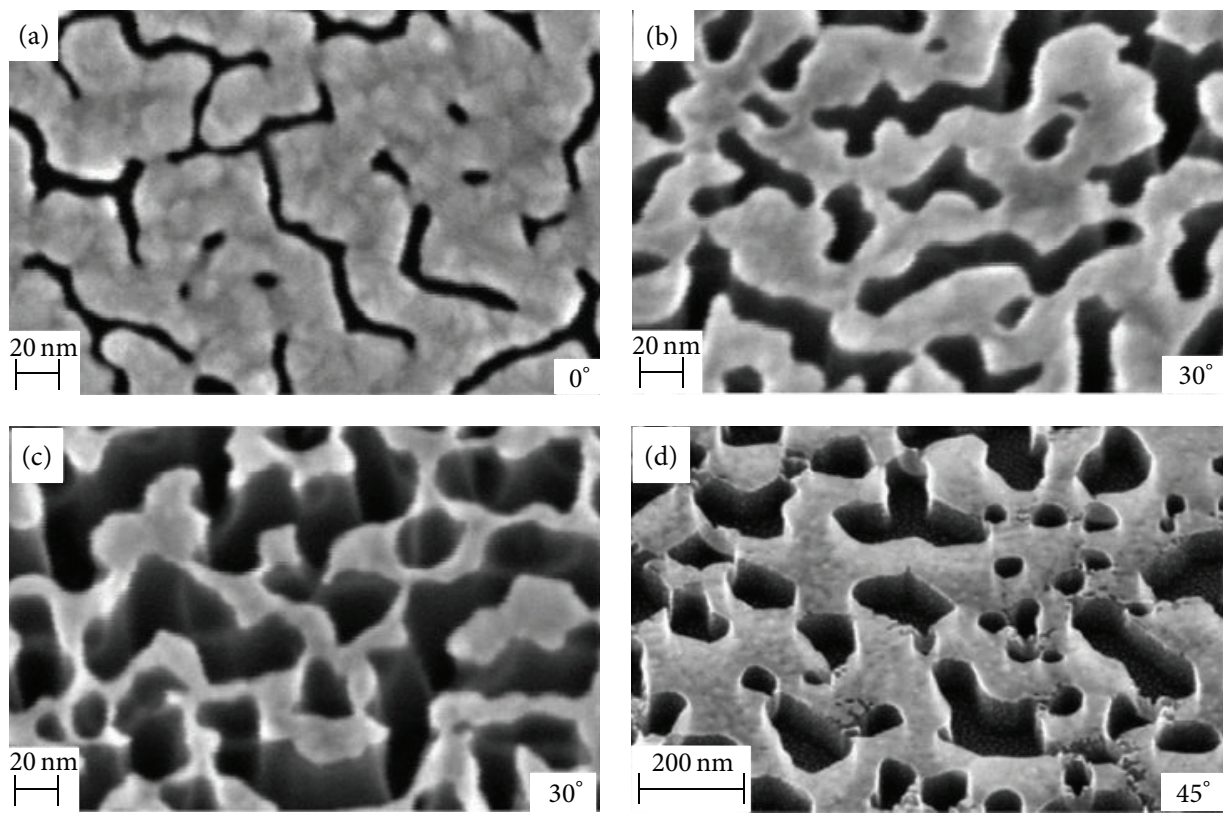

FIGURE 5: SEM images of nanstructures produced by RIE: (a) Au mask, (b) and (c) the samples after etching the substrate during 20 and 25 min, respecticely, and (d) a sample of Figure 4(d) with extra nanoholes appeared in the mask. The view angles are shown.

geometry before and after annealing of silver and gold films. In the case of silver (see Figures 4(a) and 4(b)), the annealing results in larger and more round nanoislands with a narrower size distribution. In contrast, annealing of a lace-like pattern in gold makes the narrow channels in the pattern disappear, and we obtain a smooth metal film with large irregular holes (see Figures 4(c) and 4(d)).

While arrays of metal nanospheroids and nanoholes in a thin metal film have been previously extensively studied, the nanostructures of gold and silver with the geometry and 

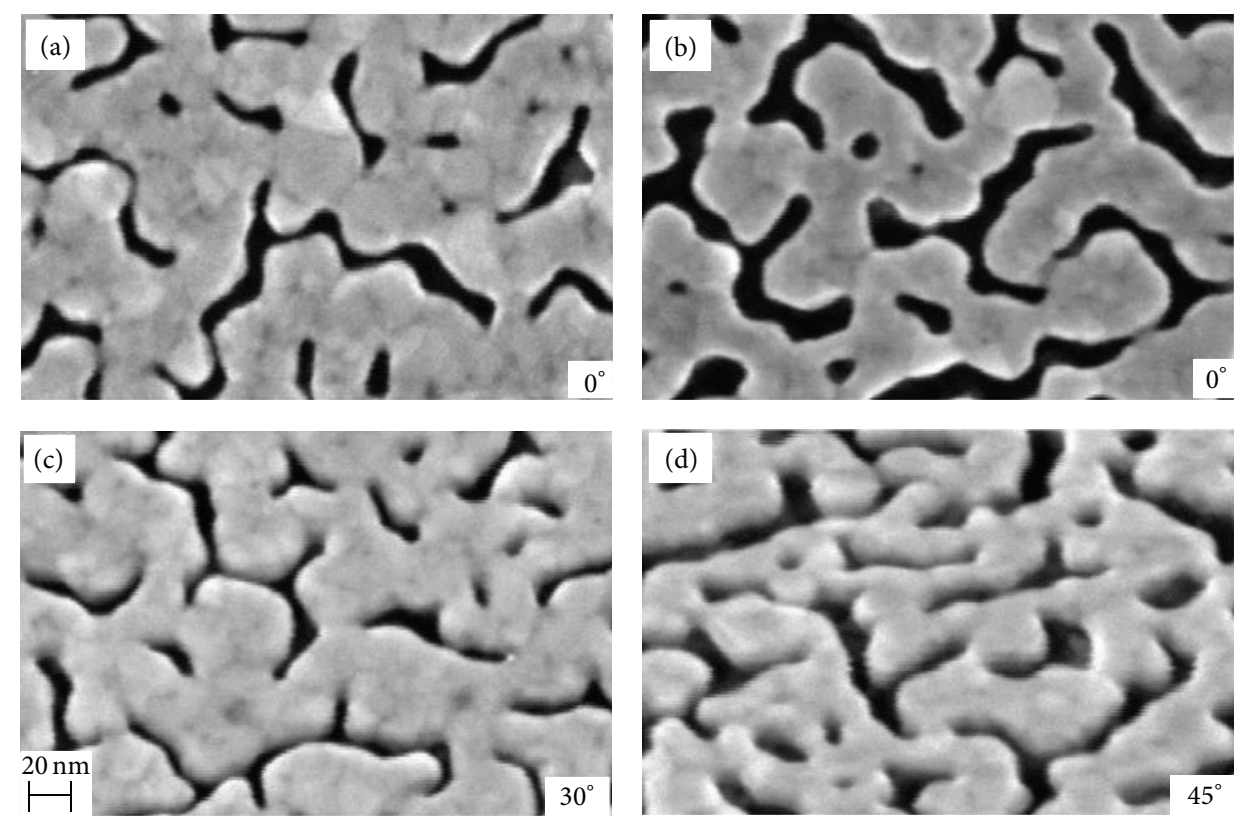

Figure 6: Plain and tilted SEM images of $16 \mathrm{~nm}$ thick Au nanostructures with a $1 \mathrm{~nm}$ thick sublayer of Ti: the structures (a) and (c) are created on the template of Figure 5(b) and the structures (b) and (d) on the template of Figure 5(c). Here $\alpha=0$. The view angles are presented.

dimensions of the patterns as in Figure 2 were not studied much. Hence, our further investigations are focused on the creation and optical characterization of this type of metal nanostructures.

The lace-like pattern of Figure 2(a) is inherent to gold. In the following, we describe a technique that can be used to fabricate such structures out of any metal. We start by creating an elevated nanostructure array (similar to a nanopillar array) by means of dry etching through the prepared gold mask (see Figure 1(b)). This mask, obtained by deposition of an $8 \mathrm{~nm}$ thick layer of gold at a rate of $1.0 \AA / \mathrm{s}$, contains depressions (in the form of narrow channels) and protrusions (see Figures 2(a) and 5(a)). The average width of the channels is ca. $7 \mathrm{~nm}$, and the width of the protrusions is about $30 \mathrm{~nm}$. The mask thickness is a critical parameter for performing RIE, because it determines the maximum obtainable etching depth. Up to a certain etching depth (say, up to $60 \mathrm{~nm}$ ), the mask pattern remains essentially unchanged (see Figure 5(b)) and the widths of the channels transferred to $\mathrm{SiO}_{2}$ are nearly equal to those in the mask. However, further etching results in widening of the channels, which can start to destroy the original mask pattern by deforming the protrusion tops (Figure 5(c)). This is caused by erosion of the gold mask during the ion bombardment. Note that the maximum sputtering yield is reached at a nonzero sputtering angle, that is, not for a normal direction of the sputtering. As a consequence, any deviation of the original mask sidewalls from vertical leads to developing a wedge of etching (an inclined mask sidewall) which, in turn, causes the mask size shrinking. The maximum etching depth of $75 \mathrm{~nm}$ was reached in 25 min of RIE (Figure 5(c)), which resulted in an increase of the channel width to about $25 \mathrm{~nm}$.
We have also used an annealed film of Figure 4(d) as an etching mask and obtained vertical, $80 \mathrm{~nm}$ high sidewalls after a 15 min processing by RIE (see Figure 5(d)). The difference of the RIE result is explained by the difference of the mask structure. Since the holes have vertical gold sidewalls, they are not subjected to the generation of the etching wedge. On the other hand, the lace-like structures have somewhat curved sidewalls, which facilitates the appearance of the fast etched planes. The etching through the hole structure exhibits one more feature. The thickness of the mask is never completely uniform, which in the SEM images is seen as darker and lighter spots distributed over the mask. In the dark spots, the metal is thinner, which can lead to appearance of additional small nanoholes in the mask during the etching procedure (see Figure 5(d)).

The etching results in elevated $\mathrm{SiO}_{2}$ nanostructures with gold residues on the top. These structures can exhibit localized surface plasmon resonances that can be used for optical applications. However, the presence of surface damages caused by RIE can destructively affect the plasmonic properties of the structures. In addition, the sizes and shapes of the metal residues are difficult to control. Therefore, we remove the metal residues from the obtained template-by selective wet etching - and obtain a pure nanostructured template in $\mathrm{SiO}_{2} / \mathrm{Si}$ or glass (Figure $1(\mathrm{c})$ ) that closely repeats the lateral geometry of the etching mask.

In the next step, the obtained template is used as a substrate for deposition of an arbitrary metal with or without an adhesion sublayer. As shown in Figure 1(d), the adhesion layer ( $\mathrm{Ti}$ ) is deposited at normal incidence (the substrate tilt angle $\alpha$ is equal to $0^{\circ}$ ). A functional layer of $\mathrm{Au}$ or $\mathrm{Ag}$ is then deposited at an angle $\alpha$ of either $0^{\circ}$ (see Figure $1(\mathrm{~d})$ ) 

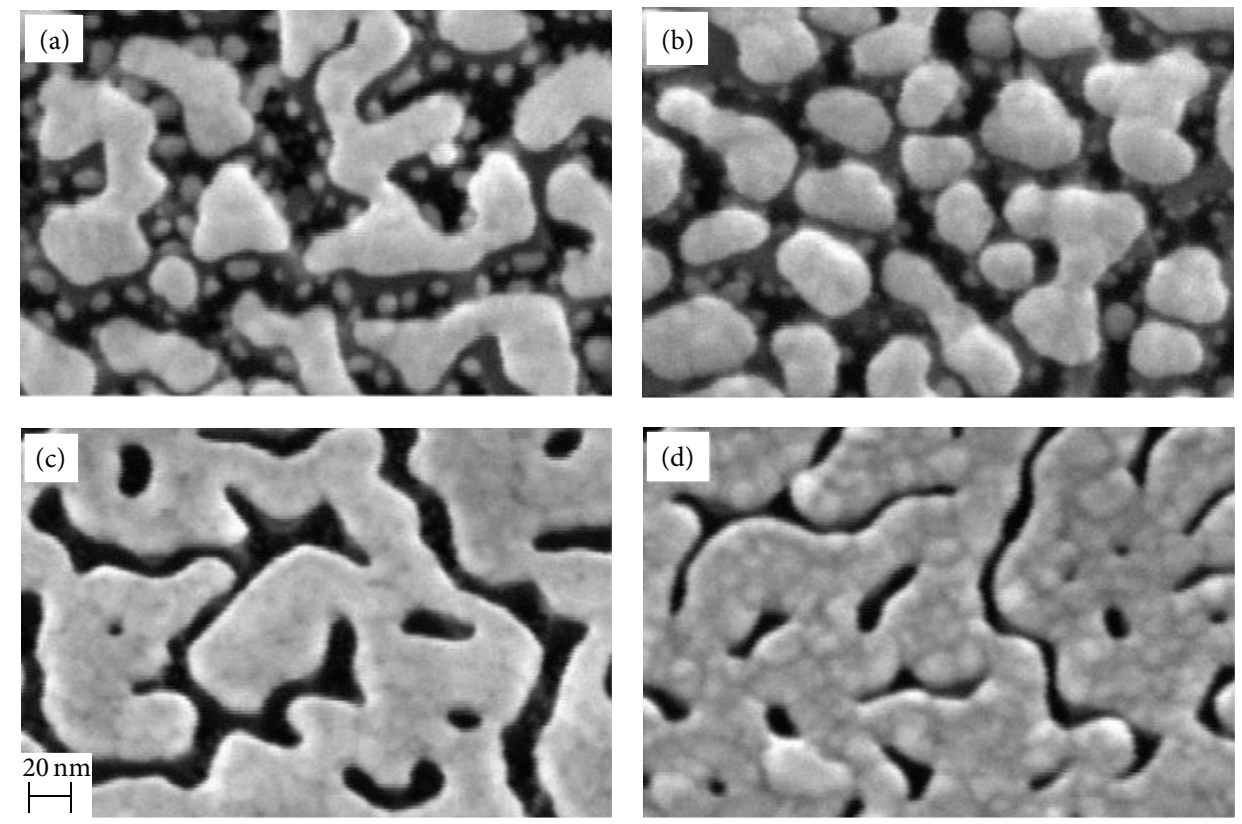

FIGURE 7: SEM images of an $8 \mathrm{~nm}$ thick nanostructures made of Au (cases (a) and (c)), and Ag ((b) and (d)) without ((a) and (b)), and with ((c) and (d)) a sublayer of Ti. In (a) and (c), $\alpha=0$. In (b) and (d), $\alpha=70^{\circ}$; the samples were rotated during the deposition. The view angle is $30^{\circ}$.

or $70^{\circ}$. In the later case, the substrate can stay motionless (Figure 1(e)) to form asymmetric nanostructures or to be rotated to obtain a uniform metal coating of the template's upper surface (Figure 1(f)). Figures 6(a) and 6(b) illustrate the results of deposition a gold films of the same thickness onto the templates of Figures 5(b) and 5(c), respectively, at normal incidence. Figures 6(c) and 6(d) show tilted views of these samples. It can be seen that evaporation of $1 \mathrm{~nm}$ thick layer of Ti and subsequent evaporation of $16 \mathrm{~nm}$ thick layer of $\mathrm{Au}$ onto the templates leads to a reduction of the average width of the channels (from 16 to $7 \mathrm{~nm}$ and from 25 to $11 \mathrm{~nm}$ for templates of Figures 5(b) and 5(c), resp.). This result is not obvious, because the deposited gold atoms arrive at the template moving along the normal to the substrate surface. To obtain some information on the mechanism of the nanostructure metallization, we consider a tilted view of the same sample (Figures 6(c) and 6(d)). The sharp contours of the template protrusions are seen to be covered with smoothly curved edges of the deposited gold layer hanging down over the channel edges. Thus, the vertical growth of the gold film is accompanied by a lateral one, despite the unidirectional deposition of the atoms. Presumably, this originates from surface diffusion of atoms that, during the deposition, receive an excess kinetic energy on the surface. If an adhesion layer is not used, the deposited metal tends to form nanospheroids on the top and sidewalls of the template protrusions.

The influence of a $\mathrm{Ti}$ sublayer on the nanostructure morphology was studied further by fabricating two more samples, in which an $8 \mathrm{~nm}$ thick layer of either $\mathrm{Au}$ or $\mathrm{Ag}$ on $\mathrm{Ti}$ is deposited at normal incidence onto the template of Figure 5(c). The pure gold nanostructures (shown in Figure 7(a)) do not exactly follow the template pattern geometry, in contrast to the $\mathrm{Ti} / \mathrm{Au}$ structures shown in Figure 7(c). Instead, they are broken into shorter segments and surrounded by many small nanoclusters. This implies that, on the top surfaces of the template protrusions, gold tends to migrate toward the centers of the isolated areas. On the other hand, the protrusion sidewalls are also favorable places for gold cluster nucleation, but due to a limited amount of the arrived gold, these clusters are very small in size. In the case of deposition of Au onto Ti (Figure 7(c)), the surface diffusion of the gold atoms is significantly reduced and, as a result, the film grows uniformly. The growth rate of the film is slightly higher at the edges of the protrusions than on the top, which leads to formation of a rim at the edges. This rim shadows the sidewalls and partly the bottom surfaces of the channels from coating by the metal. Simultaneously it reduces the channel widths. The lateral growth rate of the rim can be increased by tilting and rotating the substrate during the deposition.

Figures 7(b) and 7(d) show the lace-like Ag and Ti/Ag structures, in which an $8 \mathrm{~nm}$ thick film of silver is formed at a deposition angle of $70^{\circ}$ on a rotating template. The templates were identical for these two films and they were similar to the one shown in Figure 5(b). The substrate tilting and rotation result in more reduced average width of the channels in the Ti/Ag film (Figure 7(d)) compared to what was obtained in the Ti/Au film (Figure 7(c)). We remind that the latter film was deposited at normal incidence. Silver islands on the template without $\mathrm{Ti}$ (Figure 7(b)) are seen to have more 


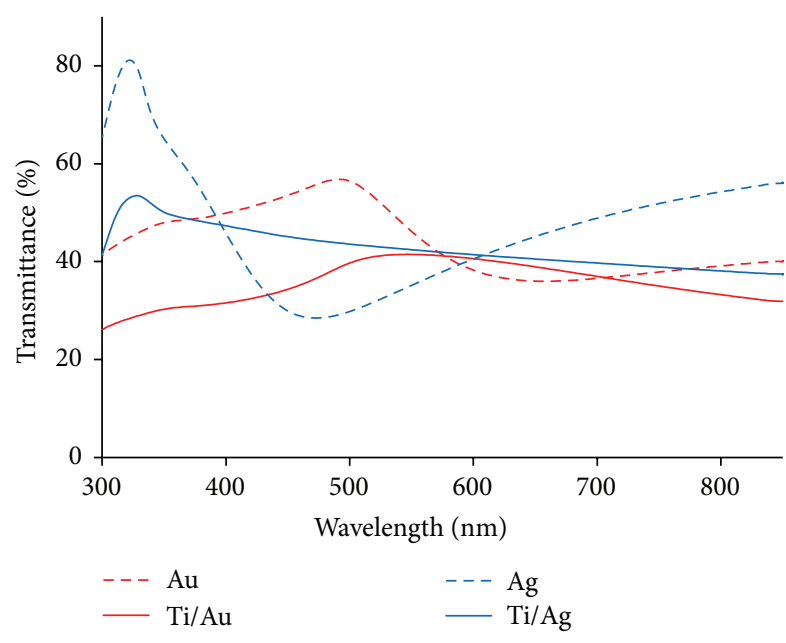

(a)

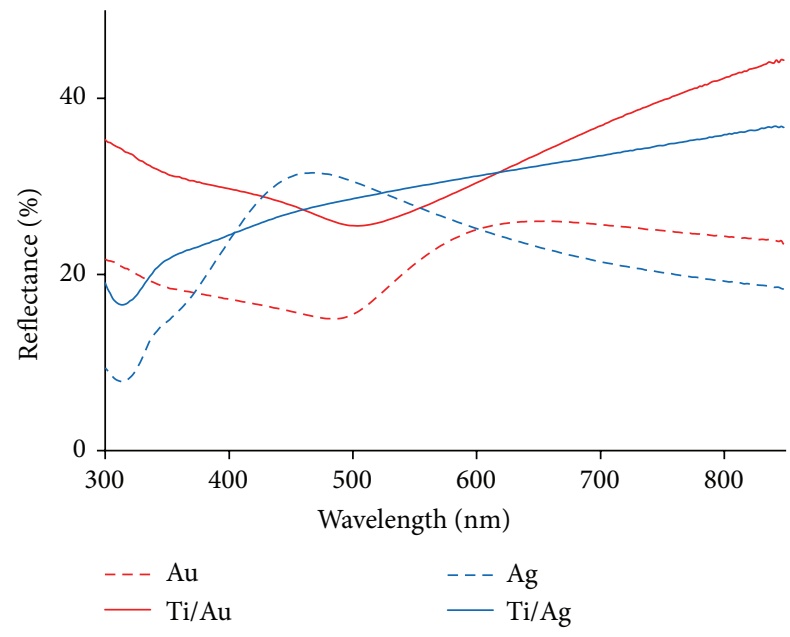

(b)

Figure 8: Transmittance (a) and reflectance (b) spectra of $8 \mathrm{~nm}$ thick $\mathrm{Au}$ nanostructures with and without Ti on glass. The metal is deposited at $\alpha=70^{\circ}$ (the samples were rotated during the deposition).

rounded edges and a narrower size distribution than the gold islands (Figure 7(a)). The samples with Ti as before accurately repeat the template geometry.

3.2. Optical Properties of Lace-Like Metal Nanostructures. The novel lace-like gold and silver nanostructures described in the above section are of interest in view of optical applications, in particular in optical spectroscopy, since they are likely to exhibit surface plasmon resonances (SPRs). To prepare a template suitable for spectroscopic analysis of the structures, we have etched an optically transparent glass substrate through a self-assembled gold mask, during $16 \mathrm{~min}$ in the RIE reactor. The etching resulted in the creation of $65 \mathrm{~nm}$ deep channels in the substrate. Then, we coated some of the substrates with an adhesion layer of $\mathrm{Ti}$ and deposited an $8 \mathrm{~nm}$ thick layer of the metal of interest (gold or silver) at different tilt angles of the substrate. The geometries of the structures are similar to those illustrated in Figure 7. In order to compare the optical properties of these samples with those of metal films on a flat substrate, we have also coated the reference glass samples with $8 \mathrm{~nm}$ thick films of gold and silver. The reference sample made of gold (silver) has a quite similar lace-like (spherical-island) geometry as the samples obtained on the etched substrates without Ti.

The optical transmittance and reflectance spectra of the fabricated gold and silver nanostructures are shown in Figure 8. The randomness in the island sizes and shapes and a strong interaction between the islands lead to spectral broadening of the overall plasmonic response of the sample $[20,23]$. The gold samples without a Ti sublayer (red dashed curves) show spectral features that refer to a Fano-type resonance [24] at the wavelengths from about 500 to $600 \mathrm{~nm}$, presumably resulting from an interplay between narrowband plasmon resonances at the edges of the structures and broadband lateral SPR excitations in the islands at longer wavelengths. We note that even though the resonance is not very sharp, the sample can still be expected to provide a high local enhancement of the incident field at the positions of the nanoisland edges. The spectra of the nanostructures with a $\mathrm{Ti}$ adhesion layer (solid curves) differ quite significantly from those of the structures without Ti. A red shift and broadening of the maximum transmittance band can be explained by the influence of $\mathrm{Ti}$ that is known to increase both the real and imaginary parts of the effective refractive index and suppress plasmonic resonant excitations.

Similarly to the spectra of the samples with gold, the spectra of silver lace-like structures fabricated without a Ti sublayer exhibit pronounced resonant features. A broad and relatively strong Fano-type resonance is now observed at shorter wavelengths, between ca. 300 and $500 \mathrm{~nm}$. It is remarkable, however, that introduction of a $2 \mathrm{~nm}$ thick $\mathrm{Ti}$ layer beneath silver nearly completely flattens the spectrum, and only weak sings of short-wavelength SPRs, between 300 and $350 \mathrm{~nm}$, can still be observed in the spectra. Thus, a sublayer of $\mathrm{Ti}$ dramatically suppresses SPRs, especially at longer wavelengths.

To receive more information about the SPR excitations and spectral modifications by the $\mathrm{Ti}$ sublayer, we have evaluated also the absorptance spectra. This was done by using the equation $A=1-T-R$, where $A, T$, and $R$ are absorptance, transmittance, and reflectance, respectively. The results of these calculations are shown in Figures 9(a) and 9(b) for gold and silver nanostructures, respectively. A crucial difference can be observed between the absorptance spectra of the fabricated structures with and without the titanium sublayer (see the dashed and solid curves). For both $\mathrm{Au}$ and Ag samples, the presence of Ti leads to a decreased absorptance in the blue and an increased absorptance in the red and near-infrared spectral range. The main reason of this difference is in our opinion a significant suppression of the SPRs.

Local enhancement of optical fields by metal nanostructures cannot always be observed in the far-field transmittance and reflectance spectra. Thus, in order to reveal a possible near-filed enhancement by our lace-like nanostructures, we have used them as SERS substrates. A Raman spectrum of MB 


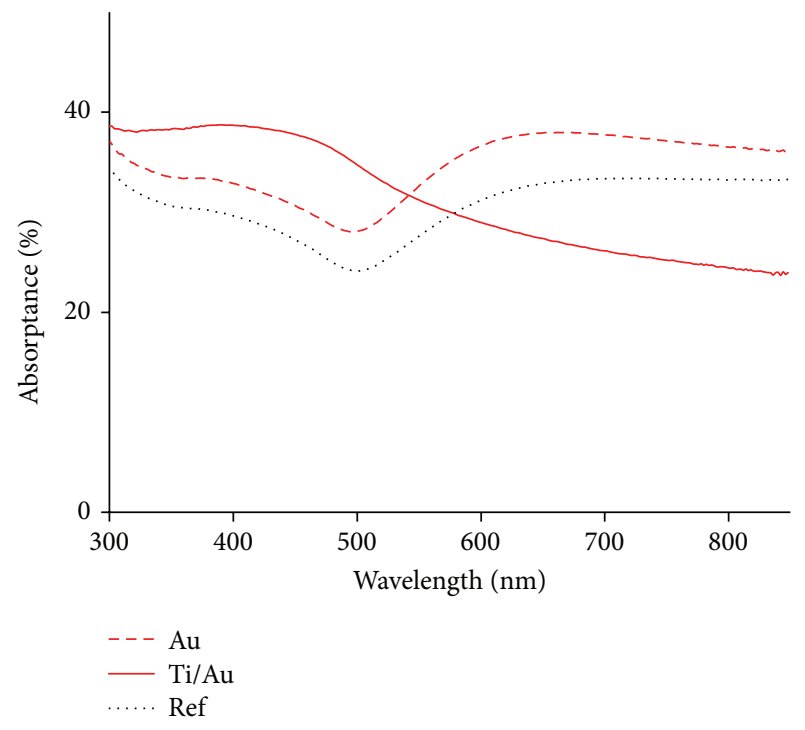

(a)

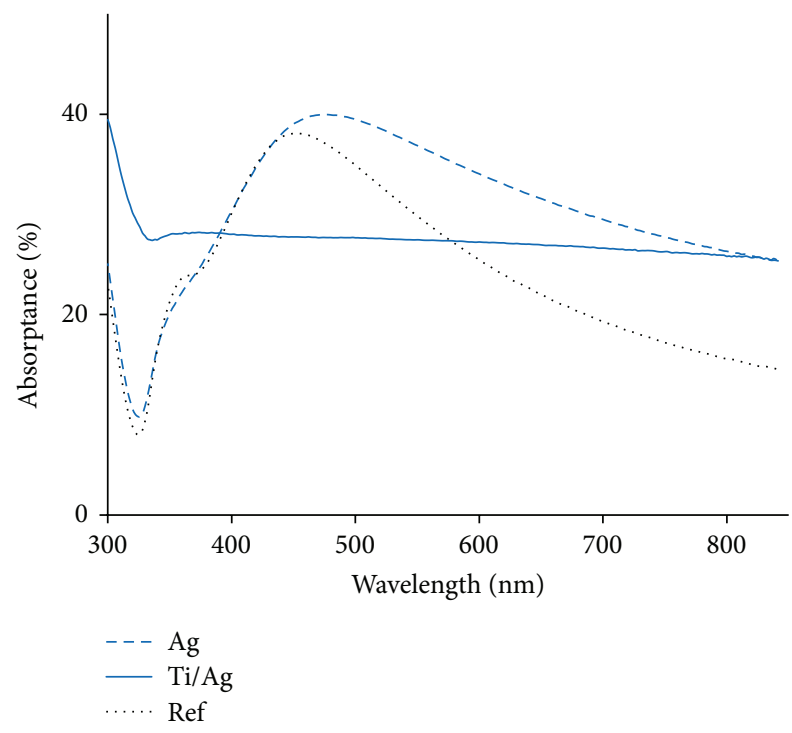

(b)

FIGURE 9: Effect of $2 \mathrm{~nm}$ thick layer of $\mathrm{Ti}$ on the absorptance of $8 \mathrm{~nm}$ thick nanostructures made of $\mathrm{Au}(\mathrm{a})$ and $\mathrm{Ag}(\mathrm{b})$ on glass. The deposition angle is $\alpha=70^{\circ}$. The samples were rotated during the deposition. "Ref" stands for an $8 \mathrm{~nm}$ layer of gold on glass, without Ti.

molecules adsorbed directly on an oxidized silicon substrate is shown by the black curve in Figure 10 (the peak at $530 \mathrm{~cm}^{-1}$ wavenumber is due to Raman scattering by crystalline $\mathrm{Si}$ ). This spectrum is used as a reference. The sample obtained by coating a nanopatterned glass template with a Ti/Ag layer ( $2 \mathrm{~nm}$ of Ti and $8 \mathrm{~nm}$ of $\mathrm{Au}$ ) shows an order of magnitude enhancement of the SERS signal (see the blue curve; the spectrum can be compared with the one reported in [25]). Thus, in spite of the smallness of our nanostructures and a significant suppression of SPRs by Ti, our structures provide a clear local enhancement of the optical near-field. It is interesting that the Raman signal can be even further enhanced

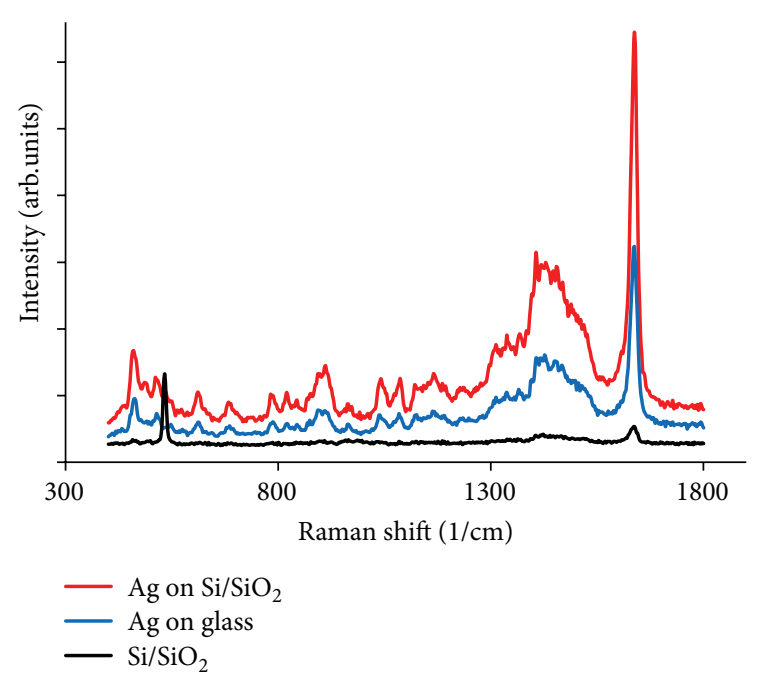

FIGURE 10: SERS spectra of $\mathrm{MB}$ adsorbed on an $8 \mathrm{~nm}$ thick $\mathrm{Ag}$ nanostructures on an oxidized $\mathrm{Si}$ wafer (red curve; the oxide thickness is $60 \mathrm{~nm}$ ) and on glass (blue curve). A $2 \mathrm{~nm}$ thick adhesion layer of Ti was used in both samples. The deposition was done at $\alpha=$ $70^{\circ}$ with rotation. The black curve is the spectrum of $\mathrm{MB}$ on $\mathrm{Si} / \mathrm{SiO}_{2}$.

by using an oxidized $\mathrm{Si}$ as a template material instead of glass. The red curve in Figure 10 shows a SERS spectrum of MB measured on such a nanostructured $\mathrm{Si} / \mathrm{SiO}_{2} / \mathrm{Ti} / \mathrm{Ag}$ sample. The additional enhancement can have the origin in the fact that the interface between $\mathrm{Si}$ and $\mathrm{SiO}_{2}$ reflects a part of the transmitted light back to the nanostructures. This reflected optical field makes an additional contribution to the Raman signal [26].

\section{Conclusions}

We have proposed and demonstrated a technique for fabrication of random metal nanostructures with the characteristic size on the order of $10 \mathrm{~nm}$ on a large-area dielectric substrate. The technique is based on self-organization of metal nanoislands with poor adhesion to the substrate. These islands are used as a mask for etching the substrate material. Afterwards, the etched substrate is directionally coated with any metal of interest to create similar nanoislands out of this metal. Using this technique, it is easy to fabricate the samples with and without an adhesion layer between the metal and the substrate. The adhesion layer makes the nanostructures mechanically stable and, therefore, they can be integrated in a more complicated device or used as plasmonic sensors for liquid or even solid analytes.

We have measured optical spectra of the fabricated lacelike nanostructures made of gold and silver and studied the influence of Ti sublayer on their properties. The presence of a very thin adhesion layer (e.g., of $2 \mathrm{~nm}$ thickness) was observed to significantly suppress the SPR excitations in the structures, and, in addition, to increase optical absorption. In spite of this, the fabricated Ti/Ag nanostructures considerably enhanced the Raman transitions of methylene blue, which 
refers to a considerable local enhancement of the light intensity by the structures. The described fabrication technique can be optimized and further developed towards specific applications in science and technology, for example, in optical sensing and surface enhanced scattering and absorption spectroscopy.

\section{Acknowledgments}

The authors acknowledge the financial support from the Academy of Finland (Project no134029). This research was undertaken at the Micronova Nanofabrication Centre, supported by Aalto University.

\section{References}

[1] J. N. Anker, W. P. Hall, O. Lyandres, N. C. Shah, J. Zhao, and R. P. Van Duyne, "Biosensing with plasmonic nanosensors," Nature Materials, vol. 7, pp. 442-453, 2008.

[2] X. Zhang and Z. Liu, "Superlenses to overcome the diffraction limit," Nature Materials, vol. 7, pp. 435-441, 2008.

[3] E. Cortes, L. Mochán, B. S. Mendoza, and G. P. Ortiz, "Optical properties of nanostructured metamaterials," Physica Status Solidi B, vol. 247, pp. 2102-2107, 2010.

[4] Y. F. Guan, A. V. Melechko, A. J. Pedraza, M. L. Simpson, and P. D. Rack, "Non-lithographic organization of nickel catalyst for carbon nanofiber synthesis on laser-induced periodic surface structures," Nanotechnology, vol. 18, no. 33, Article ID 335306, 2007.

[5] X. Sheng, J. Liu, I. Kozinsky et al., "Design and non-lithographic fabrication of light trapping structures for thin film silicon solar cells," Advanced Materials, vol. 23, pp. 843-8847, 2011.

[6] C. Eminian, F. J. Haug, O. Cubero, X. Niquille, and C. Ballif, "Photocurrent enhancement in thin film amorphous silicon solar cells with silver nanoparticles," Progress in Photovoltaics, vol. 19, no. 3, pp. 260-265, 2011.

[7] M. T. Rahman, N. N. Shams, and C. H. Lai, "Nonlithographic fabrication of $25 \mathrm{~nm}$ magnetic nanodot arrays with perpendicular anisotropy over a large area," Journal of Applied Physics, vol. 105, no. 7, Article ID 07C112, 2009.

[8] M. Fan, G. F. S. Andrade, and A. G. Brolo, "A review on the fabrication of substrates for surface enhanced Raman spectroscopy and their applications in analytical chemistry," Analytica Chimica Acta, vol. 693, no. 1-2, pp. 7-25, 2011.

[9] E. Galopin, A. Noual, J. Niedziółka-Jönsson, M. JönssonNiedziółka et al., "Short- and long-range sensing using plasmonic nanostructures: experimental and theoretical studies," Journal of Physical Chemistry C, vol. 113, pp. 15921-15927, 2009.

[10] X. Zhang, L. Zhang, M. Gao, W. Zhou, and S. Xie, "Highresolution nanosphere lithography (NSL) to fabricate highlyordered $\mathrm{ZnO}$ nanorod arrays," Journal of Nanoscience and Nanotechnology, vol. 10, no. 11, pp. 7432-7435, 2010.

[11] Y. Piao and H. Kim, "Fabrication of nanostructured materials using porous alumina template and their applications for sensing and electrocatalysis," Journal of Nanoscience and Nanotechnology, vol. 9, no. 4, pp. 2215-2233, 2009.

[12] S. Jayawardhana, G. Kostovski, A. P. Mazzolini, and P. R. Stoddart, "Optical fiber sensor based on oblique angle deposition," Applied Optics, vol. 50, no. 2, pp. 155-162, 2011.

[13] J. Sancho-Parramon, V. Janicki, M. Lončarić, H. Zorc, and P. Dubček, "Optical and structural properties of Au-Ag islands films for plasmonic applications," Applied Physics A, vol. 103, no. 3, pp. 745-748, 2011.

[14] V. Ovchinnikov and A. Priimagi, "Anisotropic plasmon resonance of surface metallic nanostructures prepared by ion beam mixing," in Proceedings of the 1st International Conference on Quantum, Nano, and Micro Technologies (ICQNM '07), Guadaloupe, Mexico, January 2007.

[15] B. Lahiri, R. Dylewicz, R. M. De La Rue, and N. P. Johnson, "Impact of titanium adhesion layers on the response of arrays of metallic split-ring resonators (SRRs)," Optics Express, vol. 18, no. 11, pp. 11202-11208, 2010.

[16] K. W. Vogt, P. A. Kohl, W. B. Carter, R. A. Bell, and L. A. Bottomley, "Characterization of thin titanium oxide adhesion layers on gold: resistivity, morphology, and composition," Surface Science, vol. 301, no. 1-3, pp. 203-213, 1994.

[17] H. Aouani, J. Wenger, D. Gérard et al. et al., "Crucial role of the adhesion layer on the plasmonic fluorescence enhancement," ACS Nano, vol. 3, no. 7, pp. 2043-2048, 2009.

[18] K. C. Vishnubhatla, S. Venugopal Rao, R. Sai Santosh Kumar, R. Osellame, S. N. B. Bhaktha, S. Turrell et al., "Femtosecond laser direct writing of gratings and waveguides in high quantum efficiency erbium-doped Baccarat glass," Journal of Physics D, vol. 42, Article ID 205106, 2009.

[19] R. M. Langford, "Focused ion beam nanofabrication: a comparison with conventional processing techniques," Journal of Nanoscience and Nanotechnology, vol. 6, pp. 661-6668, 2006.

[20] A. Shevchenko and V. Ovchinnikov, "Magnetic excitations in silver nanocrescents at visible and ultraviolet frequencies," Plasmonics, vol. 4, no. 2, pp. 121-126, 2009.

[21] V. Ovchinnikov and A. Shevchenko, "Large-area arrays of pillarbased metal nanostructures," in Proceedings of the 3rd International Conference on Quantum, Nano and Micro Technologies (ICQNM '09), pp. 125-129, Cancún, Mexico, February 2009.

[22] G. Gupta, Y. Nakayama, K. Furuya, K. Mitsuishi, M. Shimojo, K. Kajikawa et al., "Cross-sectional transmission electron microscopy and optical characterization of gold nanoislands," Japanese Journal of Applied Physics, vol. 48, Article ID 080207, 2009.

[23] V. Ovchinnikov and A. Shevchenko, "Morphology and surface plasmon resonances of silver nanocomposite layer-by-layer films," Journal of Nanoscience and Nanotechnology, vol. 9, no. 6, pp. 3872-3876, 2009.

[24] B. Luk'Yanchuk, N. I. Zheludev, S. A. Maier et al., "The Fano resonance in plasmonic nanostructures and metamaterials," Nature Materials, vol. 9, no. 9, pp. 707-715, 2010.

[25] R. R. Naujok, R. V. Duevel, and R. M. Corn, "Fluorescence and Fourier transform surface-enhanced Raman scattering measurements of methylene blue adsorbed onto a sulfur-modified gold electrode," Langmuir, vol. 9, no. 7, pp. 1771-1774, 1993.

[26] A. Shevchenko, V. Ovchinnikov, and A. Shevchenko, "Largearea nanostructured substrates for surface enhanced Raman spectroscopy," Applied Physics Letters, vol. 100, Article ID 171913, 2012. 

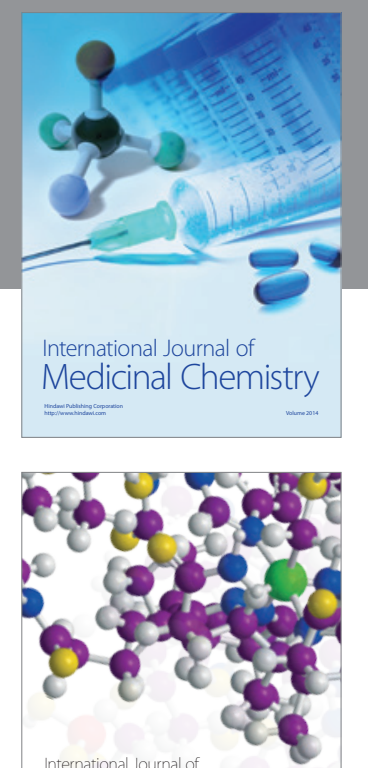

\section{Carbohydrate} Chemistry

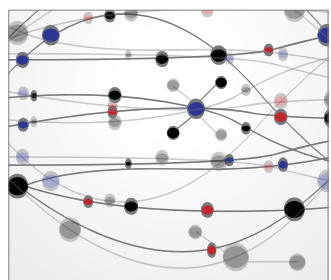

The Scientific World Journal
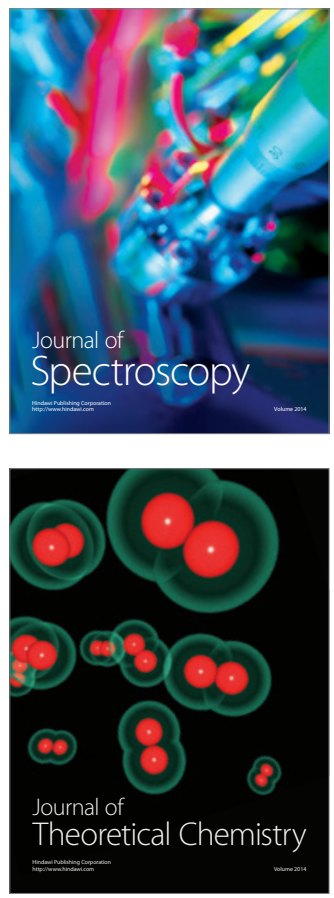
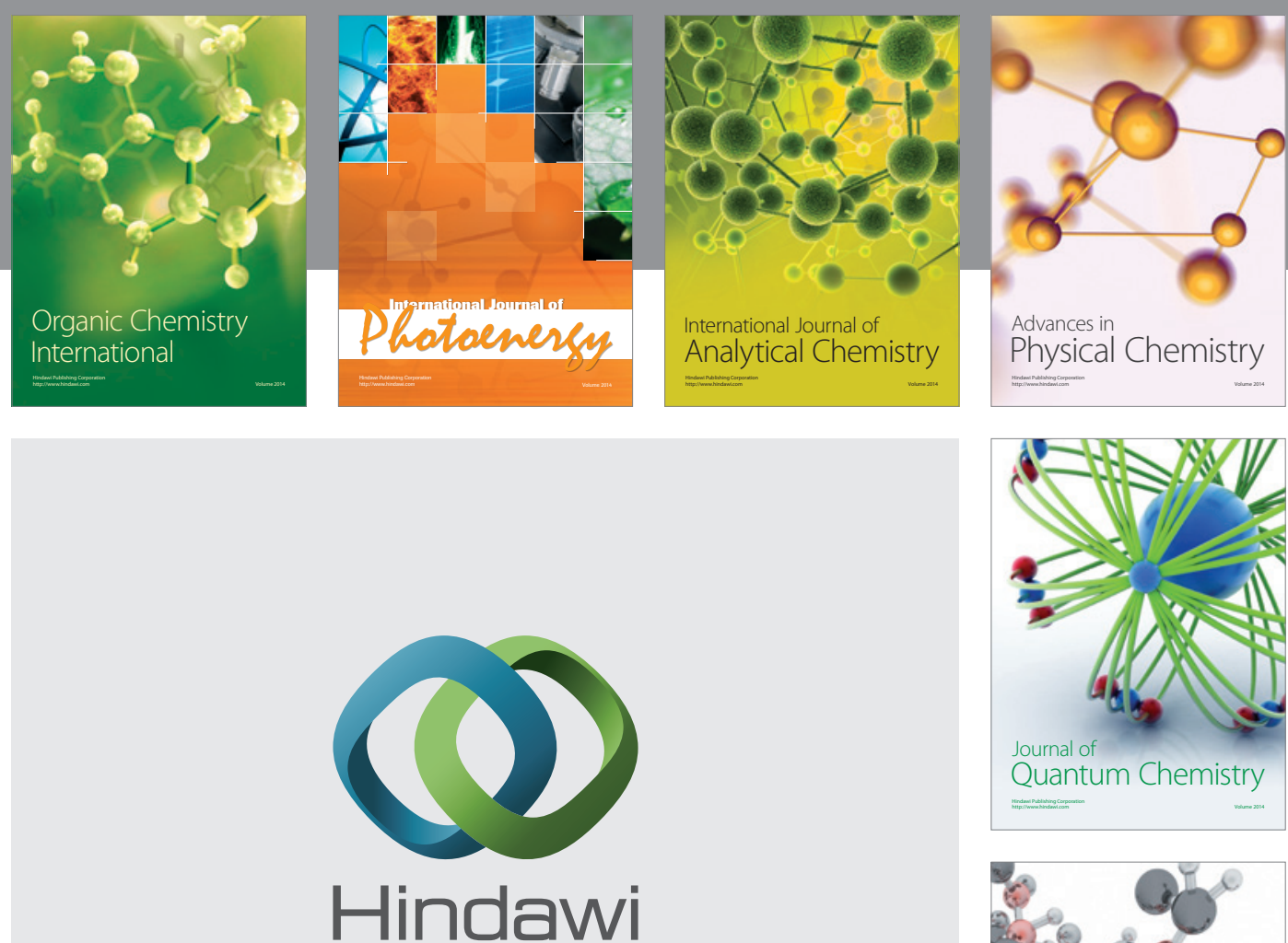

Submit your manuscripts at

http://www.hindawi.com

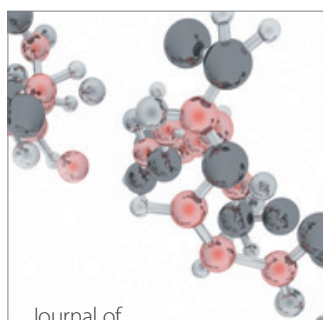

Analytical Methods

in Chemistry

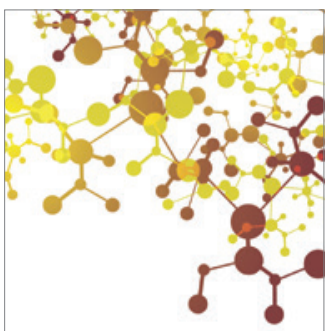

Journal of

Applied Chemistry

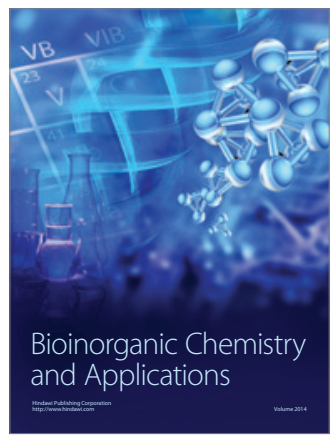

Inorganic Chemistry
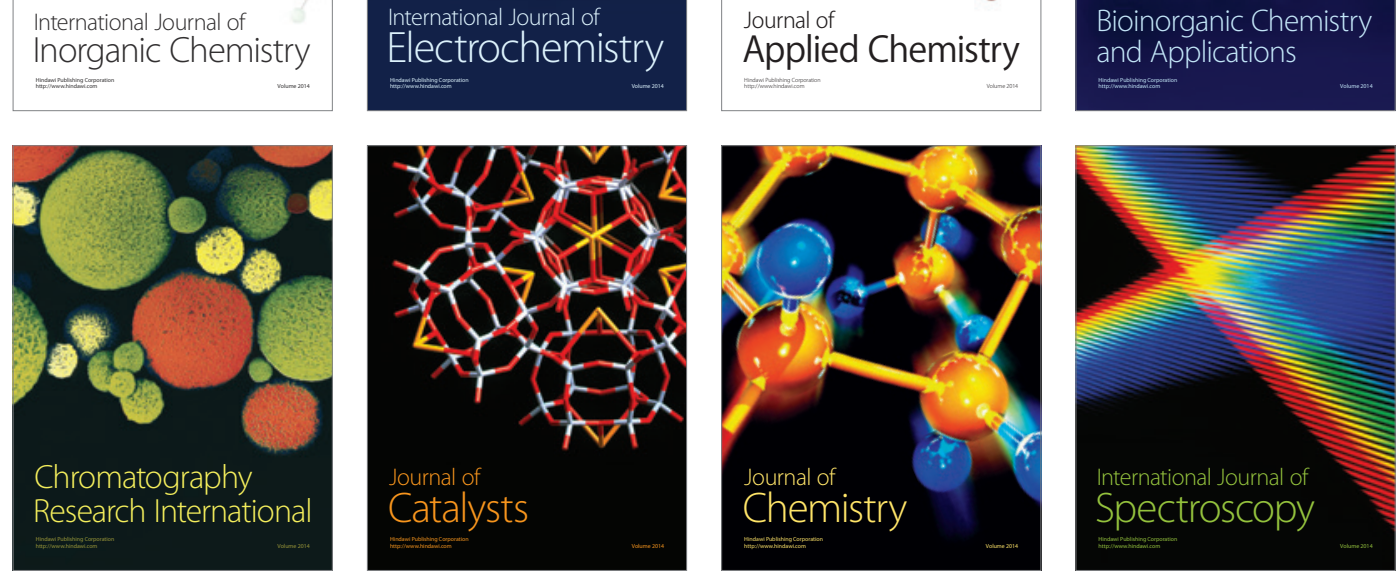